\title{
Carga inmediata de implantes insertados en alveolos postextracción. Resultados clínicos a dos años
}

\section{Immediate loading of implants inserted in postextraction sockets. A 2-year clinical follow-up}

\author{
VELASCO ORTEGA E* \\ JIMÉNEZ GUERRA $A * *$ \\ MONSALVE GUIL L $* * *$ \\ ORTIZ GARCÍA I** \\ ESPAÑA LÓPEZ A** \\ MATOS GARRIDO N**
}

\begin{abstract}
Velasco Ortega E, Jiménez Guerra A, Monsalve Guil L, Ortiz García I, España López A, Matos Garrido N. Carga inmediata de implantes insertados en alveolos postextracción. Resultados clínicos a dos años. Av Periodon Implantol. 2015; 27, 1:37-45.
\end{abstract}

\section{RESUMEN}

Introducción: El objetivo del presente estudio era mostrar los resultados del tratamiento con implantes dentales insertados después de la extracción y cargados de forma inmediata.

Método: 24 pacientes con pérdida dental unitaria o parcial fueron tratados con 50 implantes IPX (Galimplant ${ }^{\circledR}$ ) de conexión interna y de superficie arenada y grabada. Todos los implantes fueron insertados después de la extracción correspondiente y fueron cargados de forma inmediata. A los 3 meses se realizaron las prótesis definitivas.

Resultados: Los hallazgos clínicos indican una supervivencia y éxito de los implantes del 100\%. El $72 \%$ de los implantes fueron insertados en el maxilar superior mientras que el $28 \%$, en la mandíbula. Se realizaron 30 coronas unitarias y 10 puentes fijos provisionales de resina y posteriormente de cerámica a los 3 meses. Después de un periodo medio de carga funcional de 24,3 meses, no ha habido complicaciones.

Conclusiones: Este estudio indica que los implantes dentales insertados de después de la extracción y cargados de forma inmediata pueden constituir una alternativa implantológica predecible y exitosa.

PALABRAS CLAVE: Implantes dentales, implantes postextracción, prótesis inmediata, alveolos postextracción, carga inmediata, implantología oral.

\section{SUMMMARY}

Introduction: The aim of this study was to report the outcome of treatment with implant inserted after tooth extraction and immediately loaded.

Methods: 24 patients with single or partially tooth loss were treated with 50 IPX Galimplant $\circledast$ internal connection and sandblasted-etched surface implants. All implants were inserted after tooth extraction and loaded with immediate acrylic prosthesis. After a period of 3 months it realized definitive ceramic prosthesis.

Results: Clinical results indicate a survival and success rate of implants of $100 \% .72 \%$ of implants were inserted at maxilla while $28 \%$ were inserted at mandible. 30 single crowns and 10 fixed bridges immediate acrylic and after 3 months ceramic prosthesis were realized. After a mean functioning period of 24.3 months, no complications were reported.

Profesor Titular de Odontología Integrada de Adultos y Gerodontología. Director del Máster de Implantología Oral. Facultad de Odontología. Universidad de Sevilla.

** Profesor Colaborador Docente de Odontología Integrada de Adultos y de Gerodontología. Profesor del Máster de Implantología Oral. Facultad de Odontología. Universidad de Sevilla.

*** Profesor Asociado de Odontología Integrada de Adultos y Gerodontología. Profesor del Máster de Implantología Oral. Facultad de Odontología. Universidad de Sevilla. 
Conclusions: This study indicate that dental implants inserted after tooth extraction and immediately loaded may constitute a successful, predictible alternative implant treatment.

KEY WORDS: ental implants, postextraction implants, immediate loading, immediate prostheses, postextraction sockets, implant dentistry.

Fecha de recepción: 9 de septiembre 2014.

Fecha de aceptación: 13 de enero 2015.

\section{INTRODUCCIÓN}

El proceso alveolar es un tejido específico que se desarrolla conjuntamente con la erupción de los dientes. El volumen del proceso alveolar está relacionado con la forma y tamaño de los dientes, su eje de erupción y su inclinación espacial. Por lo tanto, cuando se pierden los dientes, el proceso alveolar sufre un proceso de atrofia. La cicatrización después de la extracción dental evoluciona, generalmente sin problemas, originando la reabsorción del proceso alveolar, sobre todo en sentido horizontal, dando lugar a un menor reborde alveolar $(1,2)$. De hecho, se considera que después de un periodo de 6 meses de la extracción dental, la reducción horizontal media del reborde alveolar es aproximadamente de $3,8 \mathrm{~mm}$ y la reducción vertical de $1,24 \mathrm{~mm}(3)$.

Para intentar evitar estos cambios atróficos, se ha valorado la posibilidad de insertar implantes de forma inmediata después de la extracción dental. Esta técnica quirúrgica implantológica fue introducida hace más de 30 años por Schulte (4) que describió los primeros resultados de esta técnica con pacientes después de un seguimiento clínico de 8 años con el objetivo de reducir el número de intervenciones quirúrgicas y el tiempo de tratamiento. De esta forma, se podría utilizar el alveolo como guía de la orientación del implante y preservar el hueso alrededor de la extracción con una buena respuesta de los tejidos blandos que mejoraran la estética final $(5,6)$.

La técnica de inserción inmediata de implantes postextracción ha sido bien documentada en estudios experimentales con animales demostrando que la inserción del implante inmediatamente después de la extracción dental no previene totalmente la reabsorción del hueso alveolar, sobre todo, en la pared vestibular $(7,8)$. Los hallazgos histológicos en animales han demostrado que una posición más lingual del implante en el alveolo puede reducir la reabsorción vestibular ósea y exponer menos la superficie bucal del implante, siendo más importante que el relleno con biomaterial y la colocación de membrana (8). Además, los estudios experimentales en animales han demostrado que las técnicas de preservación del alveolo postextracción limitan pero no impiden los cambios relacionados con la reabsorción ósea y de los tejidos blandos (9).

Desde un punto de vista clínico, la técnica de implantes inmediatos postextracción se ha convertido en una técnica implantológica rutinaria con un alto grado de predictibilidad similar a la inserción de implantes de forma convencional con unas tasas elevadas de éxito superior (10). Un estudio español con 22 pacientes tratados con 82 implantes insertados inmediatamente después de la extracción correspondiente confirma estos favorables resultados clínicos (11). Los implantes fueron cargados después de un periodo de cicatrización de 3 meses en la mandíbula o 6 meses en el maxilar superior. Los hallazgos clínicos indicaron un éxito de los implantes del 97,6\%. Dos implantes se perdieron durante el periodo de cicatrización. El 73,2\% de los implantes fueron insertados en el maxilar superior mientras que el $26,8 \%$ en la mandíbula. Después de un periodo medio de carga funcional de 12 meses, no hubo complicaciones tardías (11).

Además de la evaluación del éxito del tratamiento con implantes inmediatos postextracción, más recientemente, se han ido estudiando los cambios en los tejidos periimplantarios mediante parámetros clínicos y radiográficos. En este sentido, una revisión reciente muestra una pérdida ósea periimplantaria media de alrededor de 0,2 $\mathrm{mm}$ después de un año de seguimiento clínico (10). Sin embargo, algunos estudios demuestran un buen nivel de mantenimiento de los tejidos periimplantarios e incluso ganancia después de un periodo de 3-5 años $(12,13)$.

El objetivo del presente estudio era la valoración de los resultados clínicos del tratamiento con implantes mediante la técnica quirúrgica de inserción inmediata postextracción y su carga funcional inmediata. 


\section{PACIENTES Y MÉTODOS}

El presente estudio fue realizado por profesores de las unidades docentes de Odontología Integrada de Adultos y del Máster de Implantología Oral de la Facultad de Odontología de la Universidad de Sevilla.

Previamente a la realización del estudio, aquellos pacientes que padecían trastornos sistémicos graves que podían comprometer la oseointegración fueron excluidos del estudio (14). Los pacientes seleccionados eran adultos, de ambos sexos. Los pacientes autorizaron el tratamiento implantológico mediante un consentimiento informado. Antes del tratamiento, todos los pacientes fueron evaluados radiológicamente, con una ortopantomografía.

Todos los pacientes seleccionados en el estudio fueron informados de la técnica quirúrgica de inserción inmediata de implantes postextracción, así como del protocolo prostodóncico, temporalización y seguimiento, y de la posibilidad de la existencia de complicaciones y pérdida de implantes. Los criterios de éxito de los implantes fueron los recomendados por Albrektsson et al (15).

\section{Cirugía}

Todos los pacientes realizaron un tratamiento antibiótico preventivo con amoxicilina más ácido clavulánico durante una semana después de la inserción quirúrgi- ca. En los casos necesarios, cuando se presentaron dolor o inflamación, se recomendó ibuprofeno. Todos los pacientes recibieron anestesia local.

Se realizó la técnica de inserción inmediata de los implantes después de la extracción dental correspondiente que esencialmente consiste en la realización de una exodoncia lo más atraumática posible y limpieza del alveolo con una cucharilla. A continuación se realiza un fresado apical a baja velocidad (800 rpm) profundizando aproximadamente $3 \mathrm{~mm}$ para conseguir la inserción del implante con una buena estabilidad primaria. El espacio entre el implante y las paredes del alveolo no se rellenó con ningún biomaterial (Figs. 1-7).

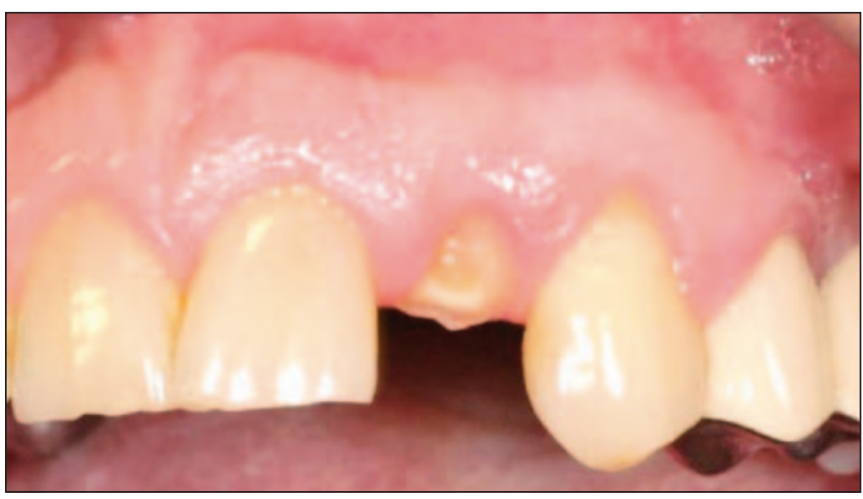

Fig. 1: Aspecto clínico del paciente con resto radicular en el incisivo superior lateral izquierdo.

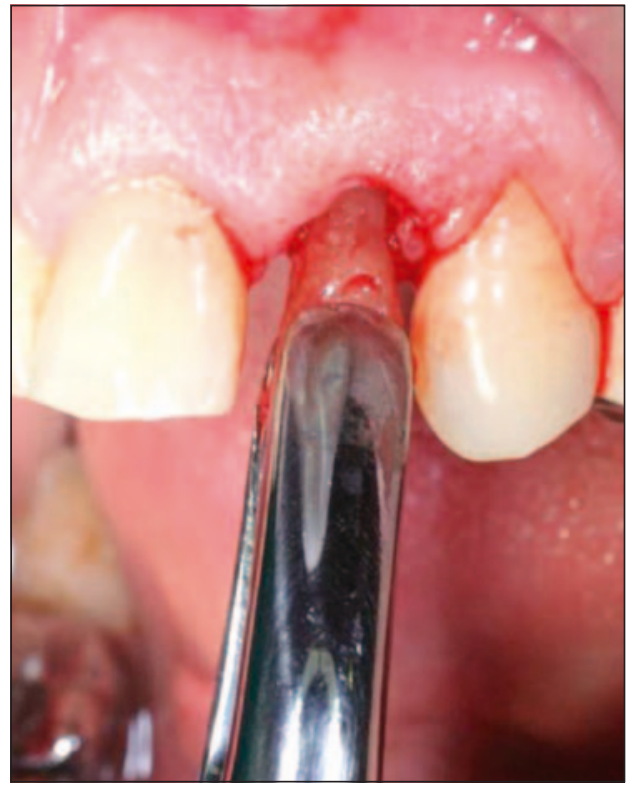

Fig. 2: Extracción del resto radicular en el incisivo superior lateral izquierdo.

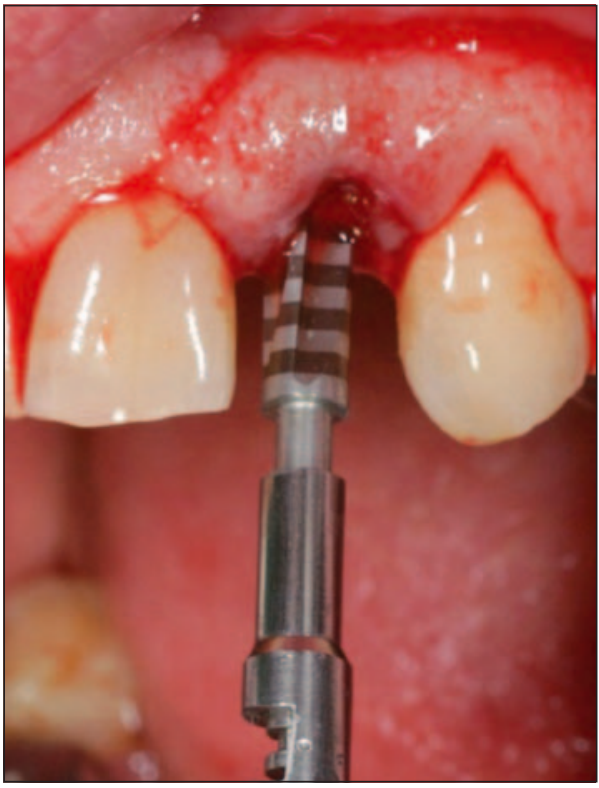

Fig. 3: Fresado apical del alveolo dentario.

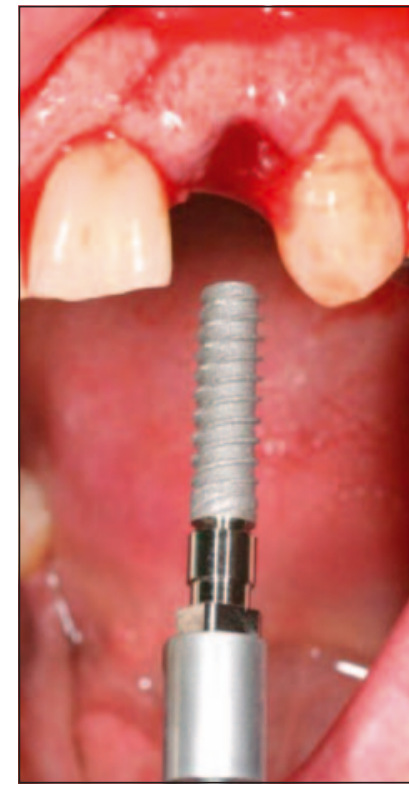

Fig. 4: Inserción de implante con diseño macroscópico roscado y espiras profundas. 


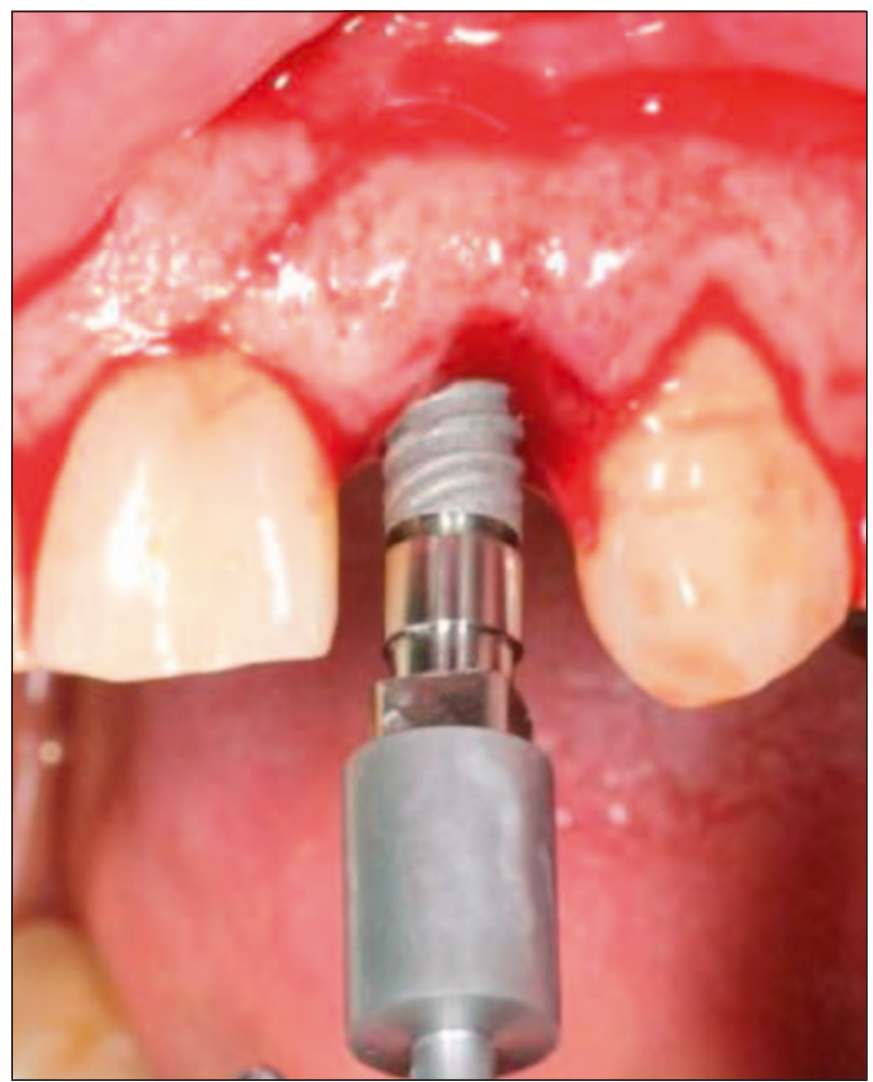

Fig. 5: Inserción de implante en el alveolo fresado.

Los implantes insertados fueron IPX® (Galimplant, Sarriá, España) roscados de conexión interna y con superficie arenada y grabada, y todos fueron estables después de la inserción. A todos los pacientes se les recomendó el enjuague diario con clorhexidina durante los primeros 30 días.

\section{Prostodoncia}

Se estableció un protocolo de carga inmediata con la colocación de las correspondientes coronas o prótesis provisionales en el mismo acto odontológico de la cirugía, y a los 3 meses la colocación de las prótesis definitivas. (Figs. 8 y 9). El tiempo transcurrido de seguimiento clínico desde la carga funcional de los implantes ha sido al menos de 24 meses.

\section{Análisis estadístico}

Se realizó una estadística descriptiva de los hallazgos clínicos del estudio, con referencia a las variables de-

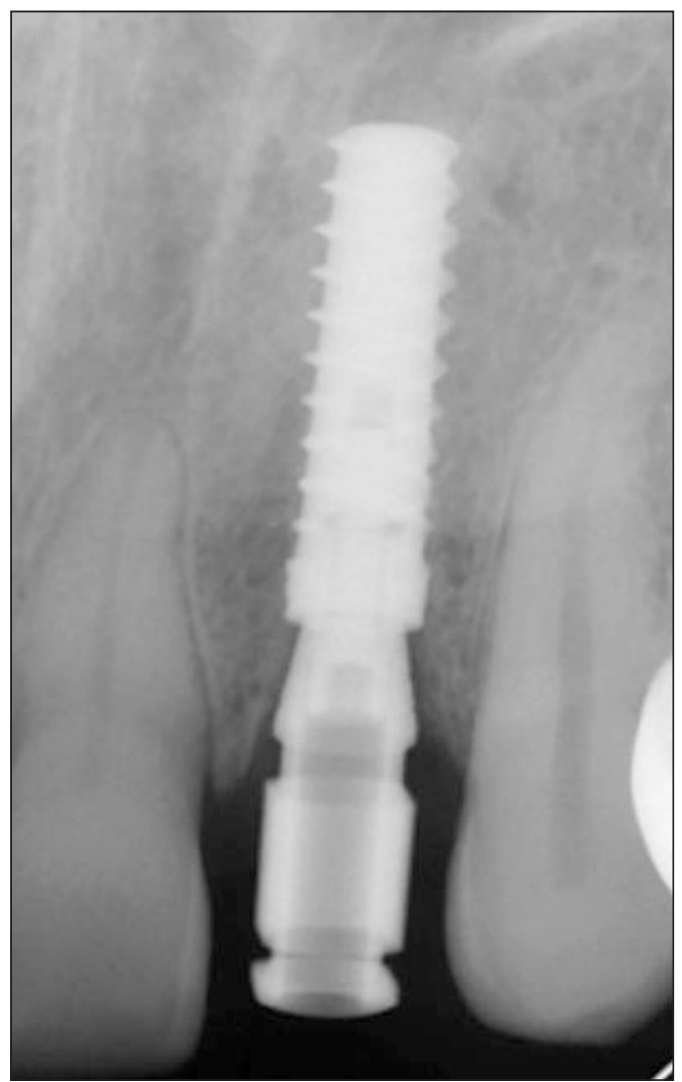

Fig. 6: Radiografía periapical del implante postextracción insertado.

mográficas de los pacientes, la inserción, supervivencia y pérdida de los implantes así como de las restauraciones prostodóncicas realizadas.

\section{RESULTADOS}

\section{Pacientes}

El presente estudio incluía a 24 pacientes con pérdidas dentales unitarias o parciales, de los cuales 12 eran hombres y 12 eran mujeres con una edad media de 50,2 años (rango: 33-63 años).

\section{Implantes}

Se insertaron un total de 50 implantes en los correspondientes 24 pacientes.

Con respecto al diámetro, se insertaron 12 implantes de $3,5 \mathrm{~mm}$ (24\%), 34 implantes de $4 \mathrm{~mm}(68 \%)$ y 4 de $5 \mathrm{~mm}(8 \%)$. Con respecto a la longitud, fueron inserta- 


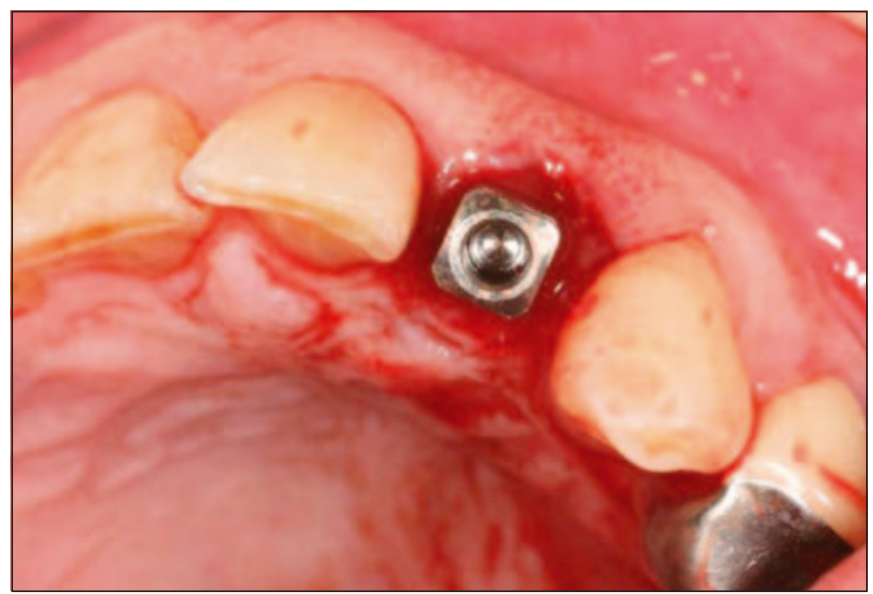

Fig. 7: Aspecto clínico oclusal del implante postextracción insertado.

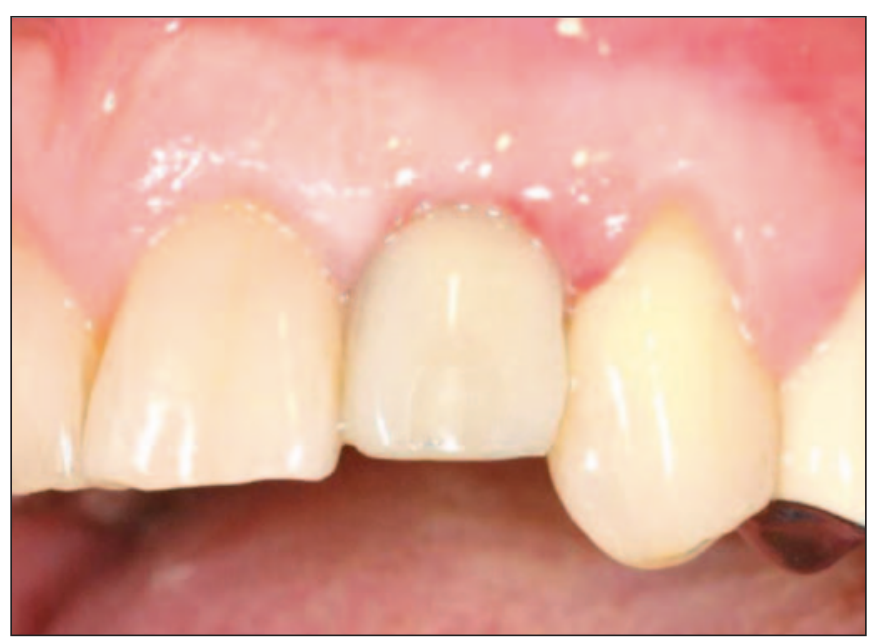

Fig. 8: Corona provisional inmediata.

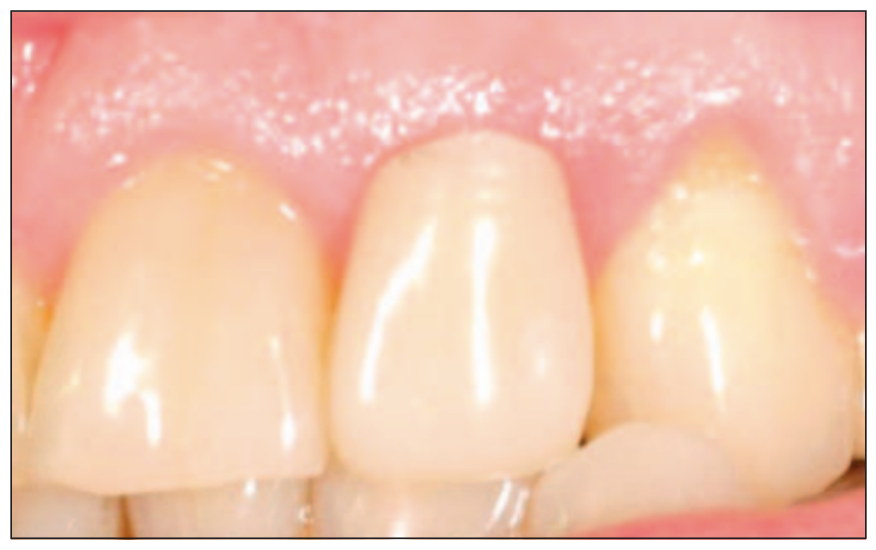

Fig. 9: Corona definitiva a los 3 meses. dos 30 implantes de $12 \mathrm{~mm}(60 \%), 12$ implantes de 14 $\mathrm{mm}(24 \%)$ y 8 implantes de $10 \mathrm{~mm}(16 \%)$.

En todos los pacientes, los implantes se insertaron de forma inmediata en el mismo acto quirúrgico de la extracción dental: 36 implantes (72\%) fueron insertados en el maxilar superior y 14 implantes $(28 \%)$ en la mandíbula; 30 implantes $(60 \%)$ se insertaron en el sector anterior (incisivos y caninos) y 20 implantes (40\%), en el sector posterior (premolares).

La pérdida media de hueso marginal fue de 0,67 mm, con un rango de 0 a $1,5 \mathrm{~mm}$.

No hubo complicaciones quirúrgicas ni prostodóncicas. Ningún implante se ha perdido durante el periodo de seguimiento clínico.

\section{Prótesis implantosoportadas}

Los 50 implantes fueron cargados de forma inmediata con las prótesis provisionales de resina (30 coronas unitarias y 10 puentes fijos con dos unidades). A los 3 meses, se realizaron las correspondientes prótesis definitivas de cerámica. El seguimiento clínico medio ha sido de 24,3 meses (rango: 24-30 meses).

\section{DISCUSIÓN}

Este estudio valora los resultados clínicos del tratamiento con implantes dentales mediante la técnica de inserción inmediata después de la extracción. Esta técnica quirúrgica se ha ido incorporando en los últimos 25 años de forma progresiva a la práctica implantológica general con buenos resultados. En este sentido, la inserción de implantes de forma inmediata después de la extracción puede favorecer una mejor cicatrización del alveolo y prevenir, aunque sea parcialmente, los cambios morfológicos derivados de la cicatrización y remodelación ósea (16-22).

Los fenómenos biológicos que ocurren después de la extracción dental y que configuran la cicatrización del alveolo y la progresiva sustitución por tejido óseo duran alrededor de 4-6 semanas, aunque la remodelación definitiva puede durar hasta 4 meses $(1,2)$. La reducción de la distancia horizontal o anchura vestíbulo lingual después un periodo de 6-12 meses después de la extracción, puede representar hasta el $60 \%$ de la anchura alveolar inicial y de alrededor del $11-22 \%$ en sentido vertical, especialmente en aquellos casos de 
extracciones múltiples que atrofian severamente el reborde alveolar $(1,2)$.

Los diversos estudios sobre implantes inmediatos postextracción valoran la respuesta de los tejidos periimplantarios después de la realización de esta técnica $(12,13,23-25)$. Se ha demostrado una reducción del hueso crestal en sentido horizontal después de la inserción de implantes, sin técnicas de regeneración (biomateriales y membranas) de forma inmediata (de 10 a $8,1 \mathrm{~mm}$ ) como a las $6-8$ semanas (de 8,8 a $5,8 \mathrm{~mm}$ ) después de la extracción, lo que indica la conveniencia de realizar una técnica inmediata para impedir una mayor pérdida de hueso (23).

En el presente estudio no se han realizado tampoco técnicas de regeneración ósea del alveolo con utilización de biomateriales y membranas, lo que confirma que en los casos de implantes postextracción unitarios, no son imprescindibles para mantener el hueso periimplantario. De hecho, un estudio reciente demuestra que la técnica quirúrgica simple postextracción sin colgajo preserva más el hueso horizontal del alveolo que cuando se utilizan biomateriales (hueso porcino y membrana de colágeno) (25).

En el presente estudio a 2 años, la pérdida media de hueso marginal fue de $0,67 \mathrm{~mm}$, con un rango de 0 a $1,5 \mathrm{~mm}$. Estos resultados son confirmados por diversos estudios que han demostrado que los implantes inmediatos postextracción mantienen de forma exitosa sus niveles originales de hueso marginal periimplantario $(12,13)$. Un estudio prospectivo a 5 años muestra una ganancia global de $0,23 \mathrm{~mm} \pm 0,43 \mathrm{~mm}$ en el nivel óseo, aunque en el primer año hubo una mínima pérdida ósea $(0,21 \mathrm{~mm} \pm 0,14 \mathrm{~mm})$. Un importante factor de éxito fue la realización de un programa supervisado de higiene oral (12). Además, los niveles de tejidos blandos pueden ser mantenidos por su importancia estética, como demuestra un estudio a 3 años en implantes postextracción maxilares donde el margen mucoso se encontraba al mismo nivel que originalmente después de la inserción en el $43 \%$ de las localizaciones bucales, mientras que en el $30 \%$ existía una ganancia de $1 \mathrm{~mm}$ (13).

Los resultados clínicos del presente estudio indican una elevada tasa de éxito con esta técnica de implantes inmediatos postextracción (100\%) que coinciden con los referidos por una revisión reciente de la literatura donde se evalúan 2.908 implantes en 46 estudios con un éxito global del $98,4 \%$ a los 2 años. Si se valora los estudios a 4 años la tasa desciende al 97,5\% (10). Es evidente que cuando se realiza una buena selección del paciente, se realiza una técnica quirúrgica cuidadosa, un protocolo adecuado prostodóncico y un programa de mantenimiento y revisiones periódicas (higiene oral) se pueden conseguir resultados clínicos muy favorables $(12,22,24)$.

En el presente estudio, en todos los pacientes se prescribió un régimen antibiótico convencional. La mayoría de los estudios relativos a la técnica inmediata postextracción indican pautas similares de antibióticos que parece sugerir mejores resultados clínicos con un menor número de fracasos de implantes $(10,11,23,25)$. La utilización de antibióticos debe ser la regla en aquellos casos de inserción inmediata de implantes postextracción en alveolo con presencia de infección o lesiones periapicales quísticas para no disminuir la tasa de éxito. Así mismo, el tratamiento antibiótico debe ser indicado en aquellas técnicas con utilización de biomateriales por defectos óseos postextracción más importantes que pudieran comprometer la inserción inmediata del implante (26-28).

En la técnica de implantes inmediatos postextracción, el diseño macroscópico de los implantes es importante para obtener una buena estabilidad primaria durante la fase quirúrgica. Un fresado más apical y una morfología con espiras amplias mejora la inserción del implante (Figs. 3-6). En la técnica de inserción inmediata postextracción, se han utilizado diferentes sistemas de implantes que han asegurado el éxito del tratamiento (11-13,22).

La superficie de los implantes es también importante porque acelera una buena respuesta ósea y favorece una más rápida oseointegración de los implantes inmediatos postextracción. Aunque no hay estudios comparativos, sí existe una tendencia más favorable en los resultados clínicos con implantes rugosos tratados con arenado y grabado ácido comparados con superficies mecanizadas $(22,24,26)$.

Los estudios han demostrado que independientemente del tipo de carga funcional realizada (convencional, precoz e inmediata), la respuesta ósea tras la inserción inmediata de implantes después de la extracción correspondiente es favorable y los resultados clínicos presentan tasas de éxito superiores al 95\% (10). En el presente estudio, se han realizado prótesis fija que después de un periodo superior de 2 años no han presentado complicaciones tardías. Un estudio realizado con 95 pacientes y 163 implantes unitarios postextracción ofrece unos hallazgos clínicos de éxito del 
$97 \%$. Las coronas unitarias fueron realizadas después de un periodo de 6 meses de la inserción de los implantes (24).

Los estudios realizados con carga inmediata en implantes inmediatos postextracción muestran que la tasa de éxito para los implantes cargados inmediatamente no difiere si habían sido insertados de forma diferida o inmediata después de la extracción o si se había realizado un protocolo de carga inmediata o precoz comparado con carga convencional (29-34). Un estudio retrospectivo compara la tasa de éxito entre implantes insertados en alveolos postextracción e implantes insertados en hueso maduro cargados de forma precoz con rehabilitaciones fijas (34). El éxito fue del 97,7\% en el grupo de inserción inmediata y del $96,6 \%$ en la inserción convencional. La pérdida global de hueso fue de 0,6 $\mathrm{mm}$ al año de carga funcional sin diferencias entre ambos protocolos (34).

Un estudio compara los resultados de la inserción de 40 implantes inmediatos postextracción en 40 pacientes (20 implantes con carga convencional y 20 implantes con carga inmediata). Después de un periodo de 24 meses, el éxito de los implantes fue del $100 \%$ en ambos grupos y no hubo diferencias significativas en la pérdida de hueso marginal que fue menor en el protocolo de carga inmediata $(1,02 \pm 0,53 \mathrm{~mm})$ que en el convencional $(1,16 \pm 0,51 \mathrm{~mm})(31)$.

$\mathrm{El}$ éxito de las restauraciones sobre implantes inmediatos postextracción no solamente está determinada por la tasa de supervivencia del implante sino, también, por otros factores como la estética de los tejidos blandos y de la restauración. En este sentido, la inserción de implantes postextracción puede favorecer la estética posterior ya que ayuda a preservar los tejidos periimplantarios, tanto mucosos como los niveles crestales óseos, aunque a veces es necesario la utilización de biomateriales o membranas (35).

\section{CONCLUSIONES}

El tratamiento con la carga inmediata de implantes postextracción puede representar una técnica implantológica con un elevado éxito clínico que ayude a prevenir los cambios atróficos en el reborde alveolar. La combinación de una cirugía adecuada puede conseguir una excelente estabilidad primaria del implante que asegura su posterior restauración funcional y estética manteniendo un buen nivel de los tejidos periimplantarios.

\section{BIBLIOGRAFÍA}

1. Van der Weijden F, Dell' Acqua F, Slot DE. Alveolar bone dimensional changes of post-extraction sockets in humans: a systematic review. J Clin Periodontol 2009;36: 1048-56.

2. Tan WL, Wong TLT, Wong MCM, Lang NP. A systematic review of post-extractional alveolar hard and soft tissue dimensional changes in humans. Clin Oral Impl Res 2012;23(suppl.):1-21.

3. Hämmerle CHF, Araujo MG, Simion M, Araujo MG. Osteology Consensus Report. Evidence-based knowledge on the biology and treatment of extraction sockets. Clin Oral Impl Res Clin Oral Impl Res 2012;23 (suppl.):80-2.

4. Schulte W, Kleineikenscheidt H, Linder K, Schareyka R. The Tubingen immediate implant in clinical studies. Dtsch Zahnärztl Zeitschr 1978;33:348-59.

5. Buser D, Wittneben J, Bornstein MM, Grütter L, Chappuis V, Belser UC. Stability of contour augmentation and esthetic outcomes of implant-supported single crowns in the esthetic zone. 3-year results of a prospective study with early implant placement postextraction.J Periodontol 2011;82:342-9.

6. Sanz M, Cecchinato D, Pjertusson B, Ferrus J, Lang NP, Lindhe J. A prospective, randomized-controlled clinical trial to evaluate bone preservation using implants with different geometry placed into extraction sockets in the maxilla. Clin Oral Impl 2010;21:13-21.

7. Araújo MG, Lindhe J. Dimensional ridge alterations following tooth extraction. An experimental study in the dog. J Clin Periodontol 2005;32:212-18.

8. Favero G, Botticelli D, Favero G, García G, Mainetti T, Lang NP. Alveolar bony crest preservation at implants installed immediately after tooth extraction: an experimental study in the dog. Clin Oral Impl 2013;24:7-12.

9. Fickl S, Zuhr O, Wachtel H, Stappert CFJ, Stein JM, Hürzeler MB. Dimensional changes of the alveolar ridge contour after different socket preservation techniques. J Clin Periodontol 2008;35:906-13.

10. Lang NP, Pun L, Lay KY, Li KY, Wong MC. A systematic review on survival and success rates of implants placed immediately into fresh extraction sockets after at least 1 year. Clin Oral Impl Res 2012;23(suppl. 5):39-66. 
11. Velasco E, Pato J, Lorrio JM, Cruz JM, Poyato M. El tratamiento con implantes dentales postextracción. Av Perio Impl Oral 2007;19(Supl.):35-42.

12. Botticelli D, Renzi A, Lindhe J, Berglundh T. Implants in fresh extractions sockets: a prospective 5-year followup clinical study. Clin Oral Impl Res 2008;19:1226-32.

13. Sanz M, Cecchinato D, Ferrus J, Salvi GE, Ramseier C, Lang NP, Lindhe J. Implants placed in fresh extraction sockets in the maxilla: clinical and radiographic outcomes from a 3-year follow-up examination. Clin Oral Impl Res 2014;25:321-7.

14. Buser D, von Arx T, ten Bruggenkate C, Weingart D. Basic surgical principles with ITI implants. Clin Oral Impl Res 2000; 11 (suppl 1):59-68.

15. Albrektsson T, Zarb G, Worthington P, Eriksson AR. The long-term efficacy of currently used dental implants: a review and proposed criteria of success. Int J Oral Maxillofac Implants 1986;1:11-25.

16. Block MS, Kent JN. Placement of endosseous implants into tooth extraction sites. J Oral MaxilloFac Surg 1991;49:1269-76.

17. Krump JL, Barnett BG. The immediate implant: a treatment alternative. Int J Oral Maxillofac Implant 1991; $6: 19-23$.

18. Gómez-Román G, Schulte W, d'Hoedt B, Axman-Krcmar D.The Frialit-2 implant system: five-year clinical experience in single-tooth and immediately postextraction applications. Int J Oral Maxillofac Implant 1997;12:299-309.

19. Lang NP, Brägger U, Hämmerle CHF, Sutter F. Immediate transmucosal implant using the principle of guided tissue regeneration. Clin Oral Impl Res 1994;5:15463.

20. Rosenquist B, Grenthe B. Immediate placement of implants into extraction sockets: implant survival. Int J Oral Maxillofac Implants 1996; 1 1:205-9.

21. Fugazzotto PA. Simplified technique for immediate implant insertion into extraction sockets: report of technique and preliminary results. Impl Dent 2002; 1 1: 79-82.

22. Morjaria KR, Wilson R, Palmer RM. Bone healing after tooth extraction with or without an intervention: a systematic review of randomized controlled trials. Clin Impl Dent Relat Res 2014;16:1-20.

23. Covani U, Bortolaia C, Barone A, Sbordone L. Bucco-lingual crestal bone changes after immediate and delayed implant placement. J Periodontol 2004;75:1605-12.

24. Covani U, Crespi R, Cornelini R, Barone A. Immediate implants supporting single crown restoration: a year prospective study. J Periodontol 2004;75:982-8.

25. Barone A, Toti P, Piatelli A, Iezzi G, Derchi G, Covani U. Extraction socket healing in humans after ridge preservation techniques: comparison between flapless and flapped procedures in a randomized clinical trial.J Periodontol 2014, 85:14-23.

26. Pato J, Jiménez A, Monsalve L, Segura JJ, Velasco E. Regeneración ósea guiada con implante unitario con nanosuperficie y betafosfato tricálcico. Av Perio Impl Oral 2010;22:127-34

27. Crespi R, Capparè P, Gherlone E. Immediate loading of dental implants placed in periodontally infected and non-infected sites: a 4-year follow-up clinical study. J Periodontol 2010; 81:1140-6.

28. Waasdorp JA, Evian CI, Mandracchia M. Immediate placement of implants into infected sites. A systematic review of the literature. J Periodontol 2010;81:801-10.

29. Cooper LF, Rahman A, Moriarty J, Chaffee N, Sacco D. Immediate mandibular rehabilitation with endosseous implants: simultaneous extraction, implant placement, and loading. Int J Oral Maxillofac Implant 2002;17:51725 .

30. Aires I, Berger J. Immediate placement in extraction sites followed by immediate loading: a pilot study and case presentation. Impl Dent 2002;1 1:87-94.

31. Crespi R, Capparè $P$, Gherlone E, Romanos GE. Immediate versus delayed loading of dental implants placed in fresh extraction sockets in the maxillary esthetic zone: a clinical comparative study. Int J Oral Maxillofac Impl 2008;23:753-8.

32. Den Hartog L, Huddleston JJR, Vissink A, Meijer HJA, Raghoebar GM. Treatment outcome of immediate, early and conventional single tooth implants in the aesthetic zone: a systematic review to survival, bone level, soft-tissue, aesthetics and patient satisfaction. J Clin Periodontol 2008;35:1073-86. 
33. Prosper L, Crespi R, Valenti E, Cappare P, Gherlone E. Five-year follow-up of wide-diameter implants placed in fresh molar extraction sockets in the mandible: immediate versus delayed loading. Int J Oral Maxillofac Impl 2010;25:607-12.

34. Peñarrocha M, Maestre L, Demarchi C, Peñarrocha D, Peñarrocha $M$. Immediate versus nonimmediate placement of implants for full arch fixed restorations: a preliminary study. J Oral Maxillofac Surg 201 1;69:154-9.

35. Sanz I, García-Gargallo M, Herrera D, Martín C, Figuero E, Sanz M. Surgical protocols for early implant placement in post-extraction sockets: a systematic review. Clin Oral Impl Res 2012;23(suppl. 5):67-79.

\section{CORRESPONDENCIA}

Prof. Eugenio Velasco Ortega

Facultad de Odontología

Avicena, s/n

41009 Sevilla

Correo electrónico:

evelasco@us.es 